\title{
Rapid Ultrastructural Changes in Leishmania donovani Amastigotes in Response to Hypo-Osmotic Stress
}

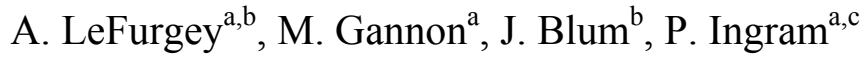 \\ ${ }^{a}$ General Medical Research Service, VAMC, 508 Fulton Street, Durham, NC 27705 \\ ${ }^{b}$ Department of Cell Biology, DUMC, Box 3709, Durham, NC 27710 \\ ${ }^{\mathrm{c}}$ Department of Pathology, DUMC, Box 3709, Durham, NC 27710
}

Leishmania donovani is a parasitic protozoan which differentiates from promastigote to amastigote on transfer from insect vector to vertebrate host. The promastigote living in the alimentary tract of the sandfly is exposed to wide variations in osmolality; it responds rapidly to hypo- or hyper-osmotic stress with changes in shape, volume, amino acid release and/or synthesis and ion content $[1,2,3]$ The overall goal of the current studies is to determine if these volume regulatory responses and their underlying mechanisms are conserved in the amastigote. This stage may be exposed to varying osmolalities in the phagolysosome of vertebrate macrophages and on release from the macrophages. In particular, these experiments examine the ultrastructural response of amastigotes to hypo-osmotic stress.

Promastigotes of $L$ donovani in log phase culture were transformed to amastigotes according to the methods of Saar et al.[4] Cells were harvested by centrifugation, washed twice in Hanks Balanced Salt Solution containing MES, 2-[N-Morpholino]ethanesulfonic acid (HBMES, pH 5.5, 37C, 330 milliosmoles), resuspended in HBMES and allowed to equilibrate for $5 \mathrm{~min}$ at $37 \mathrm{C}$. Aliquots were transferred to HBMES, pH 5.5, 37C, 330, 200 , or 100 milliosmoles for 1,4 , or 8 min prior to fixation in matching osmolalities of glutaraldehyde in sodium cacodylate buffers. Following post-fixation, embedding and sectioning, cells were examined by light and electron microscopy, and micrographs analysed using various image programs (Photoshop, NIH Image, ImagNSpect [3]).

Cells retained the original amastigote spherical shape (Figure 1) at all osmolalities and times of exposure. As early as 4 min after exposure to hypo-osmotic media, cell swelling occurred in the form of dilated nuclear envelope-endoplasmic reticulum and cytoplasmic vacuolization.

Our initial results thus indicate that, unlike the promastigote, no cell shape changes occur in the amastigote. However, these spherical cells do accommodate osmotic stress without rupture via intracellular organelle swelling and development of cytoplasmic vacuoles. Parallel biochemical studies suggest amino acid release increases as early as $1 \mathrm{~min}$. Experiments are underway using electron probe $\mathrm{x}$-ray microanalysis to determine changes in subcellular ion content which may occur also [3].

References

1. J.J Blum, Biol. Cell 87 (1996) 9-16.

2. T. Darling et al., J Protozoology 37 (1990) 493. 
3. A. LeFurgey et al., Comp Biochem Physiol A Mol Integr Physiol. 128 (2001) 385.

4. Y. Saar et al., Molecular and Biochemical Parasitology 95 (1998) 9-20.

5. Work supported by VA Merit Review Award to A.L. and by General Medical

Research and Pathology and Laboratory Medicine Services, Durham, NC, VAMC. We appreciate and thank Dr. D. Zilberstein for assistance with amastigote growth techniques and E. Whalin and W. Fennell for technical assistance.

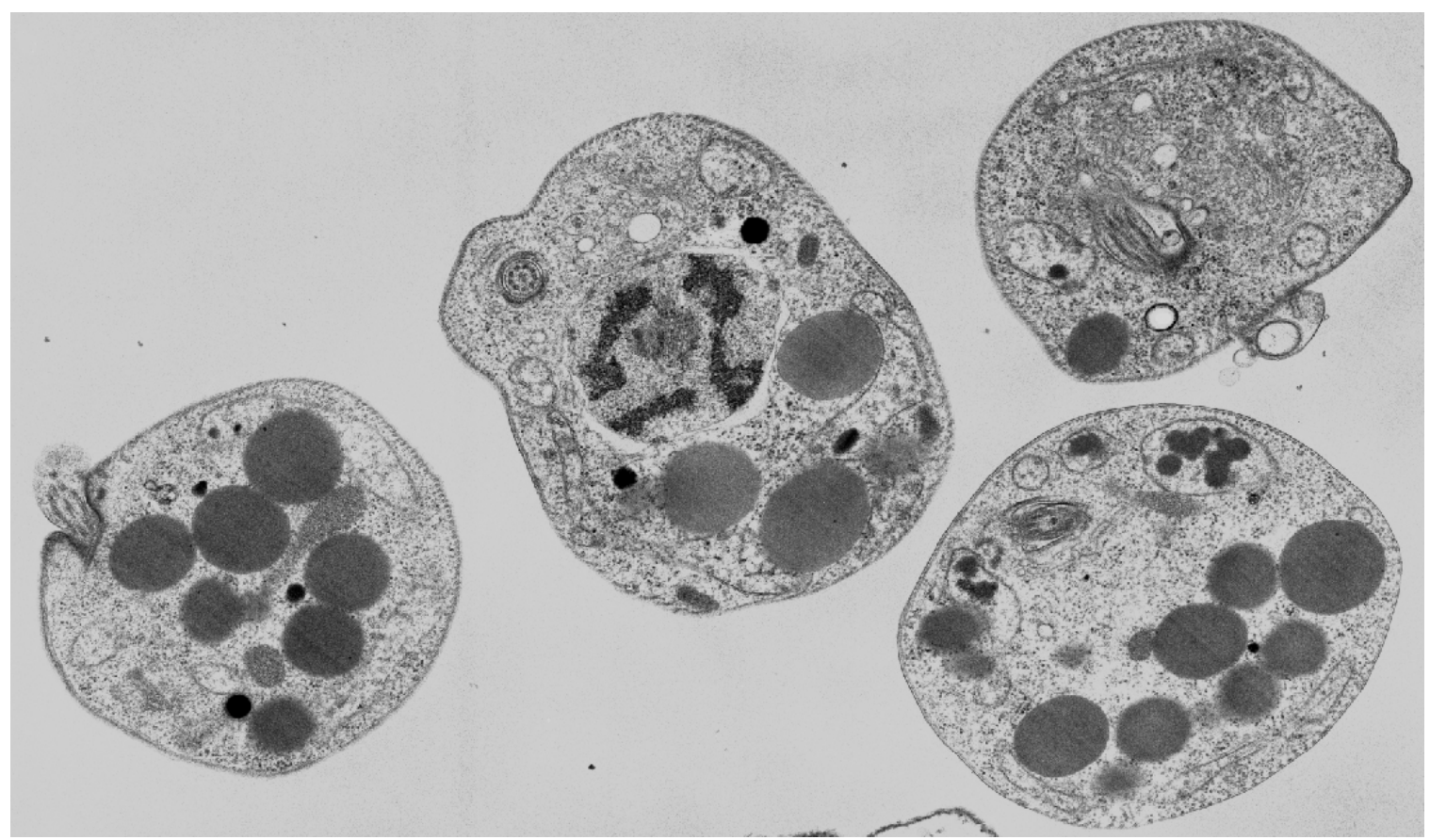

Figure 1. Transmission electron micrograph of amastigotes grown according to Saar et al. [4] at $\mathrm{pH} 5.5,37 \mathrm{C}$. Characteristic organelles including nucleus, resorbed flagellum, lipid vacuoles, mitochondria present. Image full width $=5$ microns. 